\title{
Vortex Structure in the Wall Region of an Impinging Plane Jet
}

\author{
A. Koched ${ }^{1 \dagger}$, M. Pavageau ${ }^{2}$ and F. Aloui ${ }^{1,3}$ \\ ${ }^{1}$ Ecole des Mines de Nantes, Département Systèmes Energétiques et Environnement (DSEE), \\ GEPEA, CNRS-UMR 6144, 4 rue Alfred Kastler, BP 20722-44307 Nantes cedex 03-France \\ ${ }^{2}$ Ecole des Mines d'Albi, Direction de relations internationales, Campus Jarlard, 81013 Albi Cedex 9- France \\ ${ }^{3}$ Université de Nantes, Faculté des Sciences et des Techniques - Département de Physique \\ 2 , rue de la houssinière BP 92208, 44322 Nantes Cedex 03 - France \\ $\dagger$ Corresponding Author Email: amine.koched@gmail.com
}

(Received April 25, 2010; accepted March 13, 2011)

\begin{abstract}
The work presented here comes within a research program dealing with vortex detection in the impingement region of a planar jet. In this study, experiments have been performed for a submerged turbulent water slot jet impinging normally on a flat plate, and an emphasis was put on the flow field characteristics. For this purpose, particle image velocimetry (PIV) have been employed. A comprehensive fluid mechanical data includes instantaneous and mean flow field, variance of normal and cross velocity fluctuations have been presented. The present work is also concerned with the flow structure in the impingement region where the transfers (heat/mass) occur. An attempt has been made to understand the flow structure by employing the vortex detection criteria on the instantaneous velocity vector field. Accordingly, the PIV measurements were carried out for four different Reynolds numbers: 3000, 6000, 11000 and 16000, and at three different planes: a plane parallel to the impingement plate, transverse plane of the jet and a plane perpendicular to the jet. A method of filtration, based on proper orthogonal decomposition (POD) technique was applied first to the instantaneous velocity and filtered velocity database is then used for vortex detection. Further, the results about the size, shape, spatial distribution and energy content of the detected vortices have been provided.
\end{abstract}

Keywords: Planar water jet, Impingement, Vortex, POD, Coherence.

\section{NOMENClature}

$\begin{array}{ll}a(t) & \text { temporal coefficient } \\ b & \text { Semi width of the nozzle }(\mathrm{mm}) \\ d_{i} & \text { Distance }(\mathrm{mm}) \\ e & \text { Nozzle width } \\ f & \text { Frequency }(\mathrm{Hz}) \\ \mathrm{H} & \text { Height of the jet }(\mathrm{mm}) \\ h_{i} & \text { Thickness of the impinging zone }(\mathrm{mm}) \\ I_{w}, I_{v} & \text { Turbulent intensity }(\%) \\ I_{u v} & \text { Reynolds tensor }(\%) \\ L & \text { Nozzle length }(\mathrm{mm}) \\ L_{c} & \text { Potential core length }(\mathrm{mm}) \\ \mathrm{M} & \text { Number of spatial modes } \\ \mathrm{Re} & \text { Reynolds number } \\ u, v & \text { Velocity components }(\mathrm{m} / \mathrm{s}) \\ V_{\max } & \text { Maximum velocity }(\mathrm{m} / \mathrm{s})\end{array}$

\section{INTRODUCTION}

Jet flows occur in many practical situations and are of great interest in many processes as well as in engineering applications. They are widely used for ambience separation in HVAC, fire safety (Pavageau and Gupta 2007), in food industry and in cooling of $x, y \quad$ Cartesian coordinates $(\mathrm{mm})$

\section{Greek letters}

$\Phi \quad$ Spatial function or POD mode (m)

$\lambda_{2} \quad$ Criteria for vortex detection $\left(\mathrm{s}^{-2}\right)$

$\lambda_{v} \quad$ Distance between vortices $(\mathrm{mm})$

$v \quad$ Kinetic viscosity $\left(\mathrm{m}^{2} / \mathrm{s}\right)$

Superscripts

Temporal average

Fluctuation

Subscripts

max maximum

electronic components. Despite their wide range of applications, planar jets have not been studied as extensively as axisymmetric jets. Only a few authors have put forward this configuration and examined it in detail; Beltaos and Rajaratnam (1973), Gutmark et al. (1978) and Namer and Ötügen (1988). Planar jets 
exhibit different kind of instabilities. These instabilities originate either from streamlines with strong curvature in the impingement region or due to inflection in the transverse profile of the streamwise velocity component in the lateral mixing layers. Taking into account those instabilities vortices may occur. Our work has led to the identification of these flow structures in the impingement region. Previous works on impinging air jets were performed at the GEPEA laboratory; Maurel (2001), Beaubert (2002), Gupta (2005), Abide (2005), Pavageau and Loubière (2006). These works show the presence of vortices in the impingement region and their consequence on transport phenomena between the jet and the ambiance. Large Eddy Simulations (LES) (F.Beaubert and Viazzo 2003) have shown that it is in the impingement region that mass transfer across the jet stream preferentially occurs. A lot of studies deal with heat exchange at the impingement of jets. Gardon and Akfirat (1965) studied this region in terms of heat transfer between the impinging jet and the impingement plate. Suetra et al. (1963 and 1965) explained the increase in heat transfer in the impinging region by the presence of vortices. Yokobori et al. (1983) showed experimentally, using visualization, roll vortices appearing in pairs of counter rotating rolls with their axis perpendicular to the plane of the jet. Pavageau and Loubière (2006) associated these rolls to Göertler vortices. However, Sakakibara et al. (2001) and Tsubokura et al. (2003) observed, experimentally and numerically using LES and Direct Numerical Simulation (DNS), that the twin vortices in the stagnation region are strongly related to the counterrotating streamwise vortices observed in the braid region in mixing layers and across the symmetry plane of the jet. It has been also shown that they were characterized locally by a strong vortical intensity and noticeable energy content (Loubière and Pavageau 2008). From the foregoing discussion it is clear that, there were no studies which focused on the characterization of these vortices in terms of size, center positions, vortex intensity, eccentricity and statistical distribution except the work of Pavageau and Loubière (2006) for planar air jets. It is necessary to better understand the fashion in which the large-scale structures present in the impingement region of a jet form, evolve and contribute to transfer mechanisms. The aim of our work is to detect and characterize the roll cells vortices at the impingement.

Further, the major studies reported in the literature deal with turbulent air jets. Planar water jets have received scant attention. Taking into account that the kinematic viscosity of water is smaller than air, at the same Reynolds number, it is easier to detect phenomena such as vortices. Phenomena can be observed at lower velocities making it possible to record signals with standard frequency bandwidths. Hence, it was thought desirable to undertake a systematic experimental study for confined planar submerged water slot jet impinging on a flat plate. PIV measurements have been performed in water for four different Reynolds numbers: 3000 , 6000,11000 and 16000 . In order to detect vortices near the impingement, the instantaneous flow fields for different measurement planes were post processed first by employing POD decomposition. The analysis of the most energetic POD modes shows some specific zones with high level of energy. Later, the $\lambda_{2}$ criterion (Jeong and Hussain 1995) has been applied to expose vortices from the flow. They are then characterized in terms of size, shape, intensity, etc. Further, statistical analysis was also performed for all the recorded data.

\section{EXPERIMENTAL FACILITY}

\subsection{Specifications}

A schematic diagram of the experimental setup is shown in Fig. 1. The experimental facility consists of a rectangular tank with submerged rectangular slot nozzle at the bottom having the width $e$ equal to $20 \mathrm{~mm}$. The water pumped from another tank and discharged through this nozzle forms a planar jet flowing upward.

The jet impinges vertically on a flat plate fixed at a distance $\mathrm{H}=200 \mathrm{~mm}$ from the nozzle (Fig. 2). The idea behind this is to set the geometrical aspect ratio (i.e. ratio of the height $\mathrm{H}$ of the jet and the nozzle width e) equal to $H / e=10$, which is consistent with the earlier work reported from our laboratory. Further, at this ratio turbulent intensity at the jet axis is maximal comparing to other ratios (Maurel 2001).

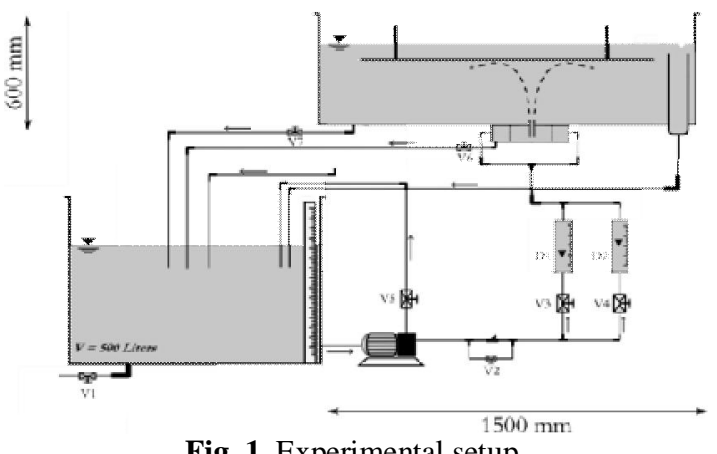

Fig. 1. Experimental setup

The ratio for the span of nozzle $\mathrm{L}=400 \mathrm{~mm}$ to the width of the nozzle e was set to $L / e=20$ to preserve the bidimensionality of the flow.

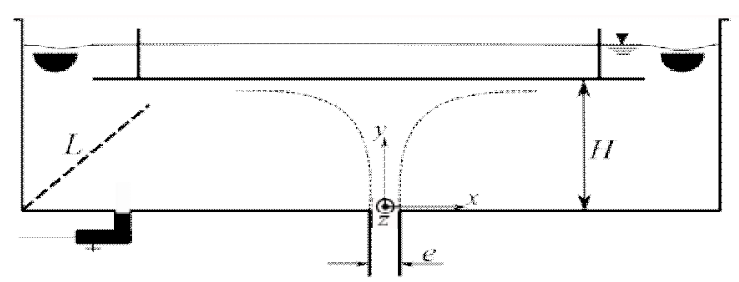

Fig. 2. Characteristic distances of the jet

The Reynolds numbers $\operatorname{Re}_{\text {buse }}=V_{\max } e / v$ were calculated using the exit velocities $\left(\mathrm{V}_{\max }\right)$ at the nozzle, width of nozzle (e) and kinetic viscosity of water $(v)$. The range of the Reynolds numbers has been chosen consistently with the works of Gupta (2005) and Yokobori et al. (1983). They mentioned that the Reynolds number strongly influences the mean and fluctuating characteristics of the jet when it is less than 6000 .

So, in order to verify the consistency of their results in case of planar water jet, the study is performed for the wide range of Reynolds number. These Reynolds number cover three situations: under limit value 
$\mathrm{Re}=3000$, the limit value $\mathrm{Re}=6000$ and values greater than limit $\mathrm{Re}=11000$ and $\mathrm{Re}=16000$ which corresponds to flowrates of $5,10,15$ and $20 \mathrm{~m} 3 / \mathrm{h}$. The corresponding velocities at the exit of the nozzle respectively are: $0.2,0.3,0.55$ and $0.8 \mathrm{~m} / \mathrm{s}$.

\subsection{PIV Measurements}

The PIV experiments were performed using a LaVision system for two different measurements planes of the jet: longitudinal and transverse plane. The schematic of the measurement arrangement of PIV is shown in Fig. 3.
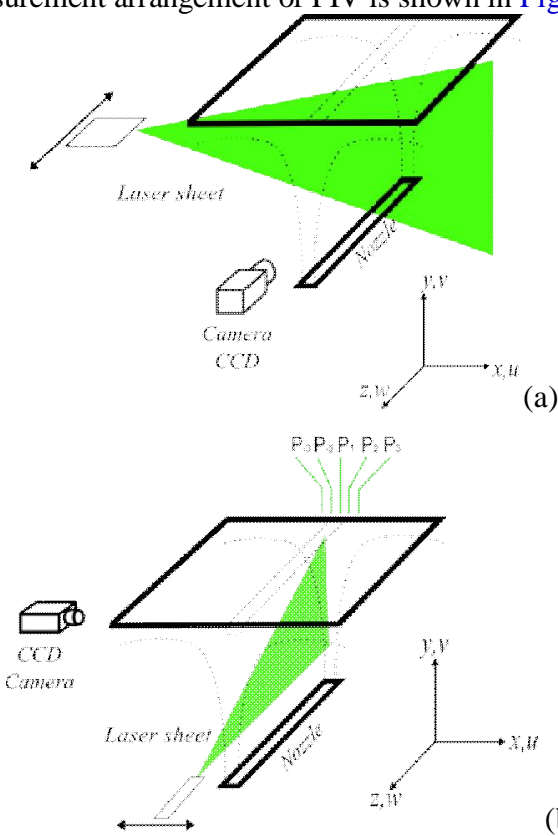

(b)

Fig. 3. Measurement planes considered for PIV technique: a) Transverse plane ; b) Longitudinal plane

The laser source adopted was a double pulsed Nd-Yag laser that had power $125 \mathrm{~mJ} /$ pulse and produces laser beam of $532 \mathrm{~nm}$ wavelength. It was synchronized with the camera using a synchronizer. The laser excites polyamide tracer particles (diameter equal to $20 \mu \mathrm{m}$ ) in the flow. A multiframe camera, with CCD sensor resolution of $1600 \times 1200$ pixels divided into small interrogations areas of $16 \times 16$ pixels, detects the light reflected by particles at two consecutives positions and instants. An adaptative algorithm based on crosscorrelation technique permits to calculate the vector field associated to the displacement of the different particles in the field of view. To increase the number of vectors, we use overlapping areas (mostly 50\%) in the two directions. The acquisition frequency of the camera is set to $15 \mathrm{~Hz}$. The time duration of recorded flow is 60 seconds. The first configuration (Fig. 3a) is considered for characterization of the flow and the second one (Fig.3b) to examine the impingement region specifically.

\section{FLOW CHARACTERIZATION}

Many authors reported interesting phenomena about the influence of Reynolds number on the development of the jet: expansion and velocity fluctuations. Tailland $e t$ al. (1967) have shown that for a range between 8500 and 38000 , Reynolds number was not affecting the development of the jet. However, Namer and Ötügen (1988) have shown that the expansion rate of the jet is mainly affected by the Reynolds numbers between 1000 and 7000 and they proposed a limit value for the Reynolds number which was 6000 . The same limit was also proposed by Maurel (2001), in study of planar air impinging jet with the important findings, that the influence of the Reynolds number still important in velocity fluctuations for $\mathrm{Re} \geq 7000$.

\subsection{Flow Characterization at the Nozzle}

Figure 4 below shows the velocity component (longitudinal and transverse) profiles at the nozzle exit for the different Reynolds number considered here: Fig.4a shows flat velocity profiles, which confirms that the flow is turbulent for all Reynolds numbers considered. Same observations were reported by Hussain and Clark (1977).

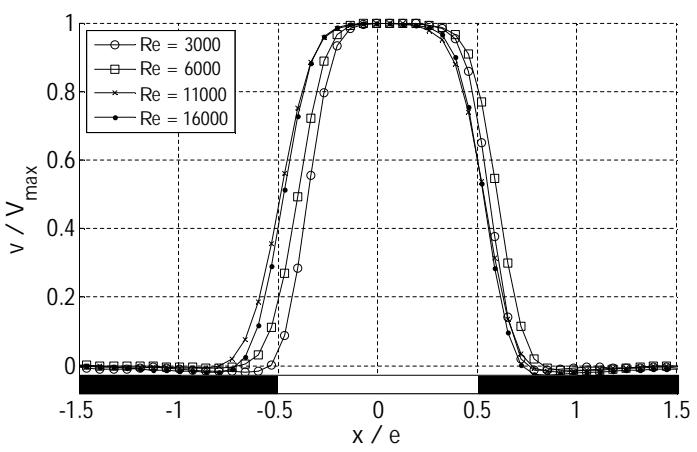

(a)

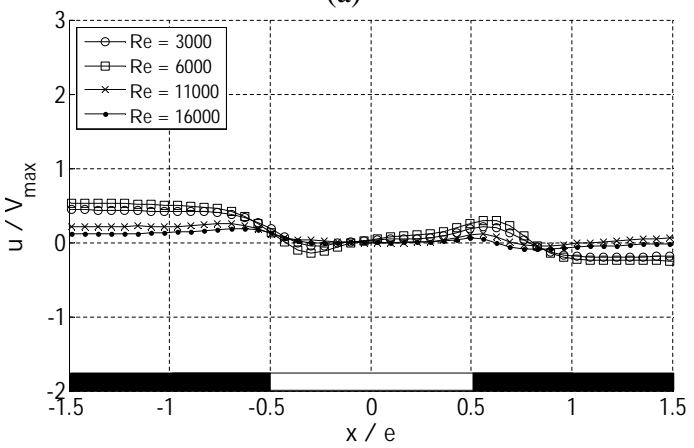

(b)

Fig. 4. Velocity profiles at the nozzle (a) longitudinalcomponent (b) transverse-component

Figure $4 \mathrm{~b}$ shows profiles of transversal velocity component $\mathrm{u}$ at $H / e=10$. From Figs. $4 \mathrm{a}$ and $4 \mathrm{~b}$ it has been also observed that the profiles of mean longitudinal and transverse velocities seem not to be affected by the variation of Reynolds number, unlike turbulent intensities and Reynolds tensor i.e. $I_{u}=\sqrt{\overline{u^{2}}} / V_{\max }, I_{v}=\sqrt{\overline{v^{2}}} / V_{\max }$ and $I_{u v}=\overline{u^{\prime}} \cdot \overline{v^{\prime}} / V^{2}{ }_{\max }$ (Figs.5 a-c). Figures 5a and 5b show the profiles of turbulent intensities $I_{u}$ and $I_{v}$ at the exit nozzle for each Reynolds number considered. The Figs. 5 a-c shows the important turbulent activities near the boundary of the nozzle which shows the starting of the mixing layers near the shear zone of the jet. The maximum of $\mathrm{I}_{\mathrm{v}}$ (Fig. 5a) reaches $17 \%$ for $\mathrm{Re}=16000$ and $8 \%$ for $\mathrm{Re}=3000$, however, the maximum of $I_{u}$ (Fig. 5b) didn't exceed $6 \%$. 


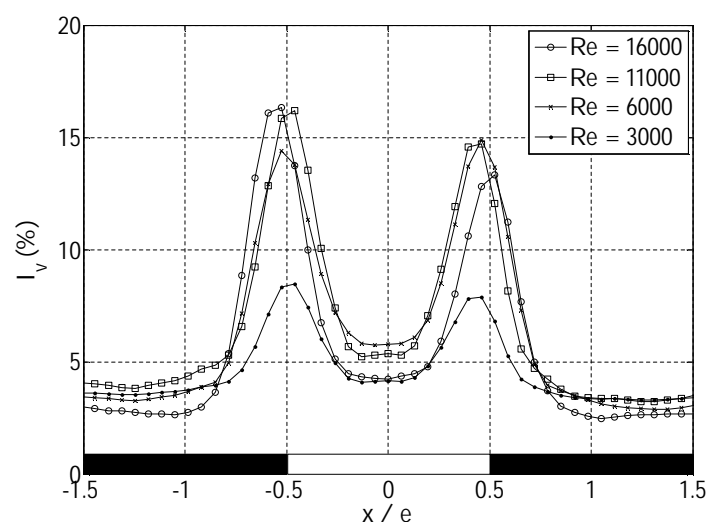

(a)

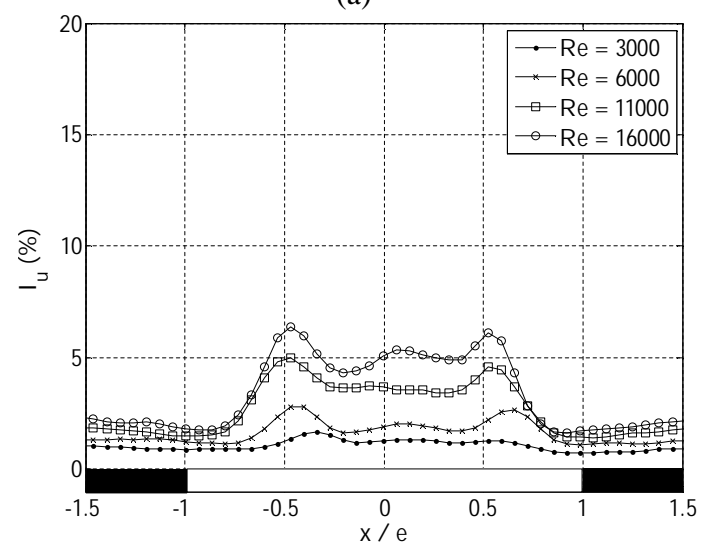

(b)

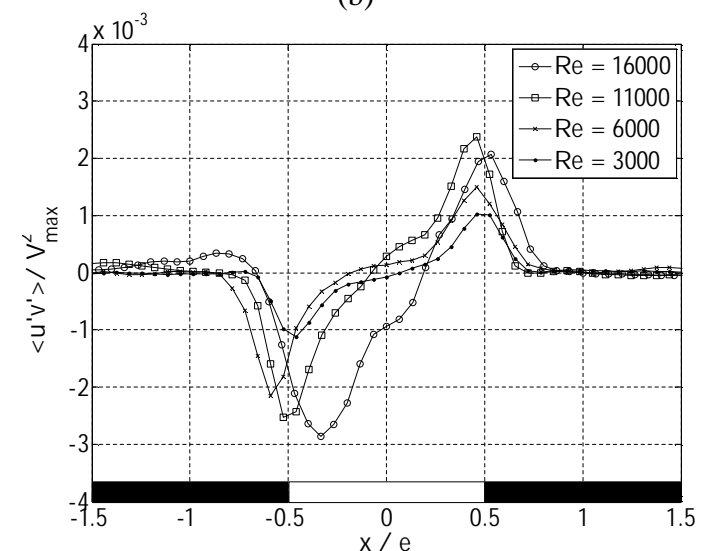

(c)

Fig.5. Turbulent intensities and Reynolds tensor at the nozzle: (a) $I_{v} \quad$ (b) $I_{u} \quad$ (c) $I_{u v}$

\subsection{Flow Structure}

Figure 6 shows an example of the streamlines calculated from the mean velocity field measured in the transverse plane of the jet for $\mathrm{Re}=16000$. It shows mainly three different regions in the jet whatever the Reynolds number considered.

The first region was observed nearly at $y / e=2$ (Fig. 6) from the nozzle in the direction of mean flow, the second region where the jet starts expanding and the third region is the impingement region from where the streamlines change directions and become tighten. Here, authors would like to put emphasize on the recirculation on both sides of the jet where the streamlines are concentric.

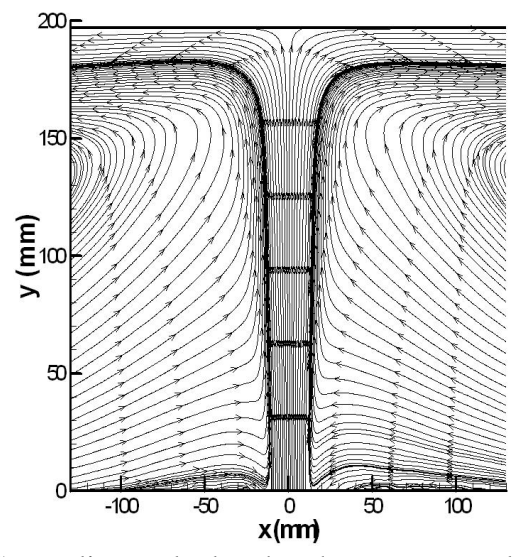

Fig. 6. Streamlines calculated at the transverse plane of the jet $(\operatorname{Re}=16000, H / e=10, L / e \geq 20)$

These three regions can be further verified by plotting the evolution of centerline longitudinal velocity (Fig.7). The first zone identified here is the potential core where the axial velocity is nearly $v=0.99 \times V_{\max }$. The potential core length has been observed $L_{c}=3 \times e$ for $\operatorname{Re} \geq 6000$ and $L_{c}=1,5 \times e$ for $\operatorname{Re}<6000$. According to literature, the potential core length was found between 3 and 5 times the nozzle width (Maurel 2005). It can be seen also that, the length of the potential core was affected for $\operatorname{Re}<6000$. This has also been observed in the past by Beltaos and Rajaratnam (1973), Rajaratnam (1976), Antonia et al. (1983), Lepicovsky (1986) and Maurel (2001).

The second zone identified is the developed region. Different empirical correlations were proposed in the literature to describe the profiles of velocity in this region.

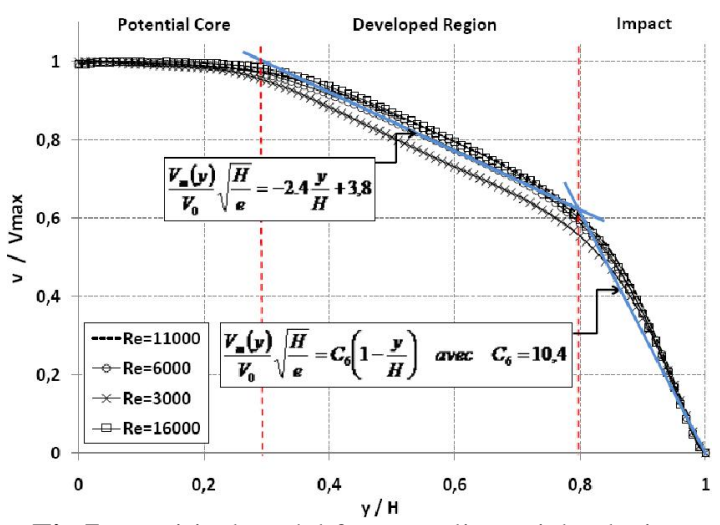

Fig.7. Empirical model for centerline axial velocity decrease of the jet $(H / e=10, L / e \geq 20)$

Beltaos and Rajaratnam (1973) proposed a given correlation:

$\frac{V(y)}{V_{\max }} \sqrt{\frac{H}{e}}=2.5 \times\left(\frac{y}{H}\right)^{-\frac{1}{2}}$

While, Maurel and Solliec (2001) and Maurel et al. (2004) proposed another correlation which gives better fit with our data: 


$$
\begin{aligned}
& \frac{V(y)}{V_{\max }} \sqrt{\frac{H}{e}}=C_{1} \frac{y}{H}+C_{2} \\
& \text { with } C_{1}=-2.9, C_{2}=4.5
\end{aligned}
$$

Nevertheless, the empirical coefficients $\mathrm{C}_{1}$ and $\mathrm{C}_{2}$ are not the same. In the present work the constant considered were found equal to $\mathrm{C}_{1}=-2.4$ and $\mathrm{C}_{2}=3.8$.

The third zone detected, is the impingement or the impact region. The centerline velocity decreases to zero at the impact. It is characterized by a significant lateral diffusion of momentum for the mean flow. Further, this region is of great interest as the significant transfers (heat/mass) appear in this region. Beltaos and Rajaratnam (1973) proposed a semi-analytical correlation for the decrease of centerline velocity in this region i.e. $0.75 \leq y / H \leq 0.98$

$$
\begin{aligned}
& \frac{V(y)}{V_{\max }} \sqrt{\frac{H}{e}}=C_{3}\left(1-\frac{y}{H}\right)^{-\frac{1}{2}} \\
& \text { with } C_{4}=5.5
\end{aligned}
$$

Later, Maurel (2005) proposed another correlation, which provides better fits with the present our experimental data:

$$
\begin{aligned}
& \frac{V(y)}{V_{\max }} \sqrt{\frac{H}{e}}=C_{4}\left(1-\frac{y}{H}\right) \\
& \text { with } C_{4}=15.5
\end{aligned}
$$

In the present study $\mathrm{C}_{4}$ was set to 10.4 .

The models proposed shows that the center line velocity is independent of Reynolds number.

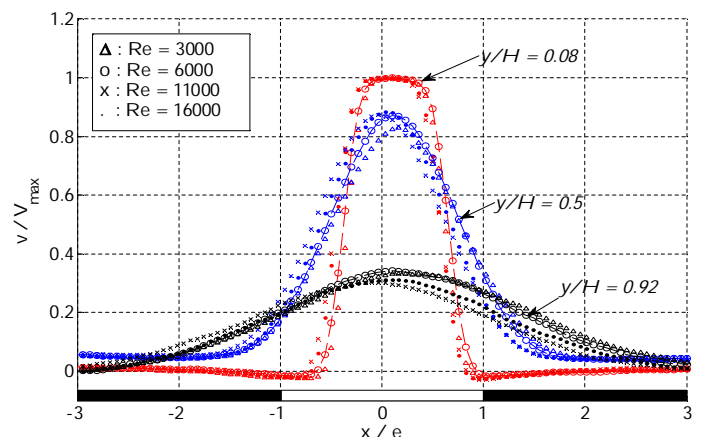

(a)

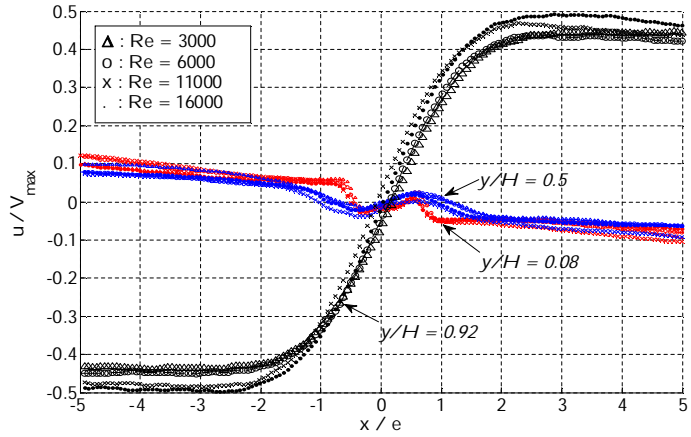

(b)

Fig.8. Transverse profiles of the longitudinal and transversal mean velocity components $v, u$

$(H / e=10$ and $L / e \geq 20)$
Again to confirm the tendency observed in Fig. 7, the profiles of longitudinal and transversal velocity components $(v, u)$ have been plotted at three different transverse positions $(y / H)$ with varying Reynolds number (Figs. $8 \mathrm{a}$ and $8 \mathrm{~b}$ ). Figures $8 \mathrm{a}$ and $8 \mathrm{~b}$ show the longitudinal and transverse velocity profiles doesn't show any variation with the Reynolds number which confirms that the development of the jet is not affected by Reynolds number.
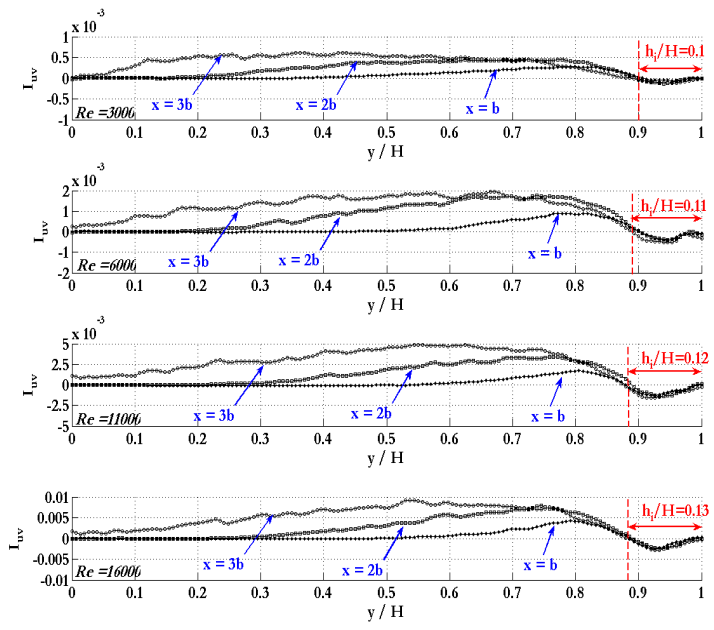

Fig. 9. Variation of $\mathrm{I}_{\mathrm{uv}}$ with Re number $(H / e=10$ and $L / e \geq 20)$

The kinematic description of the impact region of the jet has not been the subject of many studies although significant transfers occur only in this region.

The quantification and optimization of these transfers go through the understanding of mechanisms at their origin. Some works mostly in thermal heat exchange shows a link between the increase of heat transfer activity at the impingement and the longitudinal roll cells eddy structures formed at the impact of the jet (Tsubokura et al. 2003; Yokobori et al. 1983). We will especially focus in this region and on these structures in the following.

\section{THE IMPACT ZONE OF THE JET}

\subsection{Thickness of the Impact Region}

Gutmark et al. (1978) first estimated the thickness of the impact zone $\left(h_{i}\right)$ for the planar air jet, which was about $15 \%$ of the total height of the jet $(\mathrm{H})$. In the present work the thickness of the impact zone is determined from the longitudinal profiles of Reynolds stress $\overline{u^{\prime} v^{\prime}}$ at three different transverse $(\mathrm{x}=\mathrm{b}, 2 \mathrm{~b}$ and $3 \mathrm{~b})$ positions with different Reynolds number (Fig. 9). The changing in sign of Reynolds stress values for (from + to - ) reflects the starting of impact region. From Figs. 9 it has been also observed that $h_{i}$ is slightly affected by the Reynolds number. Indeed, for $\mathrm{Re}=3000$, the ratio $h_{i} / H=10 \%$ and it increases only to $13 \%$ for $\mathrm{Re}=16000$ (Figs. 9). Pavageau and Gupta (2001), and Maurel and Solliec (2001) and Maurel et al. (2004) didn't mention any influence of the Reynolds number in case of twin and simple planar air jet and reported a 


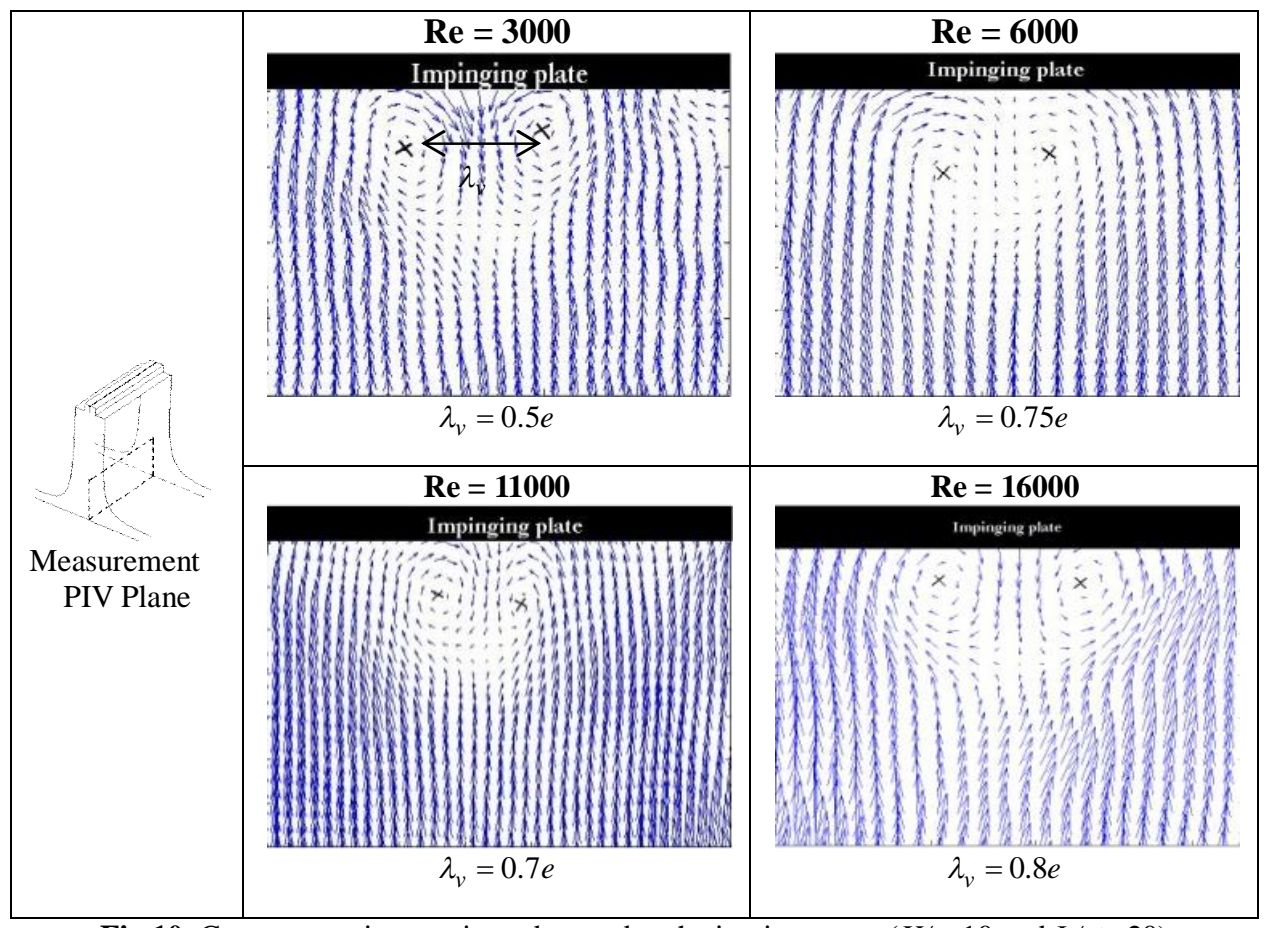

Fig.10. Counter rotating vortices observed at the impingement $(H / e=10$ and $L / e \geq 20)$

ratio of $h_{i} / H=13 \%$.

\subsection{Vortex at the Impingement Region of the Jet}

PIV measurements give the global flow field of the jet. They also efficiently provide information about the associated eddy structures in the near-wall or impact region. Hence, it is quite possible to point out the influence of the impingement on the longitudinal and transverse mean flow. Four different Reynolds numbers were considered in order to study their influence on the size of the eddy structures at the impingement region. Yokobori et al. (1983) and Sakakibara et al. (1997) underlined the presence of the longitudinal roll cells counter rotating structures in the impinging zone. They specified that these structures were due to the fact that the impingement was at the level of the transition zone of a free jet; thus, they concluded without any strong argument that these structures disappear when $H / e>15 \approx 20$.

To detect vortices at the impingement we considered PIV measurements on the mid-plane of the jet (Fig. 3b). Figure 10 shows the influence of Reynolds number on the distance between eddy structures of a same counter rotating pair of vortices $\left(\lambda_{v}\right)$. It has been observed that for $\operatorname{Re}<6000, \lambda_{v}=0.5 e$ and in the case of $\operatorname{Re}>6000$ the distance $\lambda_{v}$ was found equal to $\lambda_{v}=0.75 \times e$, Maurel and Solliec (2001), obtained a similar prediction, they found $\lambda_{v}=0.8 \times \mathrm{e}$ for $\mathrm{Re}=13500$ and $\mathrm{H} / \mathrm{e}=10$ for a planar air jet. However, this analysis is remaining based on instantaneous observations and didn't confer to these results a general aspect.

In most cases the flow structures are convected by the mean flow and couldn't be detected from the direct analysis of velocity fields. In the present work, to extract them a method of filtration based on Proper
Orthogonal Decomposition (POD) technique (Lumley et al. 1967) was applied first to the instantaneous velocity field. Filtration consists in removing from the fluctuating velocity fields the effects of perturbation on the flow (mainly due to laser reflexion, particle deposition, etc.). For that, the instantaneous velocity fields are first decomposed into $M$ spatial functions (modes), noted $\Phi^{(k)}(r)$, and temporal coefficient, noted $a^{(k)}(t)$ :

$u(r, t) \cong \sum_{k=1}^{M} a^{(k)}(t) \cdot \Phi^{(k)}(r)$

To exactly describe the velocity fields, 652 modes are necessary. A truncation to the 100 first most energetic modes (spatial and temporal) is done. The filtered velocity fields are later subjected to $\lambda_{2}$ criterion for the vortices center core detection.

\subsection{Statistical Analysis}

To detect vortices at the impingement, we considered PIV measurements on the plane $(y, z)$ of the jet (Fig.3a). This plane is located at the center of the nozzle. Vortices occur at the impingement zone, so their maximum size will not exceed the thickness of the impinging zone $h_{i}$ evaluated in $\$ 3.1$. Taking into account the variation of $h_{i}$ with Reynolds number, we consider a field size of $\mathrm{L} \times 0.2 \mathrm{H}=400 \times 40 \mathrm{~mm}^{2}$.

For statistical analysis, we considered data from PIV measurement for total established flow duration of 30 seconds.

Typically, statistical analysis shows that the total number of vortices detected is around 5500 for each 
Reynolds numbers under test: on average, this corresponds to twelve coherent structures per each instantaneous velocity vector field.

The characterization of vortices is made through the determination of the position of their center core, their sizes and shape. The $\lambda_{2}$ criterion (Jeong and Hussain 1995) was used here for the detection of the center core of the vortex. This criterion relies on the basic assumption that a vortex core center is associated with the existence of a local pressure minimum. It has an interesting property which consists in discriminating swirling and shearing motion, what is not achievable by visualizing vorticity.

To characterize geometrically the detected vortices, we examined the spatial distribution of tangential component of velocity $V_{\theta}$ around the vortex center. It is here assumed that a vortex core is marked by an increase in tangential component of velocity with radial position inside the vortex, and by a decrease in tangential component of velocity with radial position outside. Subsequently, the size and shape of a detected structure is estimated by numerically finding the maxima of $V_{\theta}$ along 8 directions. These directions are defined by the angle they form with respect to the horizontal axis $\left(\left(0^{\circ}, 180^{\circ}\right),\left(45^{\circ}, 225^{\circ}\right),\left(90^{\circ}, 270^{\circ}\right)\right.$ and $\left.\left(135^{\circ}, 315^{\circ}\right)\right)$

Four diameters are estimated for each detected vortex $D_{\alpha}\left(\alpha=0^{\circ}, 45^{\circ}, 90^{\circ}\right.$ and $\left.135^{\circ}\right)$ depending on the directions considered (Fig.12). The vortex radii defined as the distance to vortex centre from the point where, in the corresponding direction $\alpha, V_{\theta}$ reaches a maximum. From this, it is defined for each vortex:

- A mean diameter:

$D_{m}=\frac{1}{4} \sum_{\alpha=0^{\circ}, 45^{\circ}, 90^{\circ}, 135^{\circ}} D_{\alpha}$

- An eccentricity:

$E_{x}=\frac{\max \left(D_{\alpha}\right)}{\min \left(D_{\alpha}\right)}, \quad \alpha=0^{\circ}, 45^{\circ}, 90^{\circ}, 135^{\circ}$
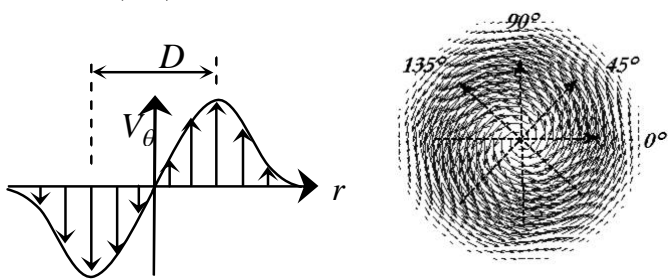

Fig. 12. (a) Tangential component velocity profile within the core of a vortex; (b) Radial directions considered for diameter estimation of the vortex

The information below are stored for each vortex detected from series of instantaneous velocity fields:

- Number of the snapshot from which the structure was detected,

- Center core coordinates,

- Diameters $D_{\alpha}\left(\alpha=0^{\circ}, 45^{\circ}, 90^{\circ}\right.$ and $\left.135^{\circ}\right)$ and eccentricity,
- Rotation direction (according to the sign of vorticity at center core of the detected vortex).

Table 1 Statistic according to number of detected vortices and their rotation direction $(H / e=10$ and $L / e \geq 20)$

\begin{tabular}{|c|c|c|c|}
\hline $\operatorname{Re}$ & $\begin{array}{c}\text { Nbr. Of } \\
\text { vortices }\end{array}$ & Clockwise & $\begin{array}{c}\text { Anti- } \\
\text { clockwise }\end{array}$ \\
\hline 3000 & 5245 & $52 \%$ & $48 \%$ \\
\hline 6000 & 5722 & $52 \%$ & $48 \%$ \\
\hline 11000 & 5746 & $50 \%$ & $50 \%$ \\
\hline 16000 & 5551 & $49 \%$ & $51 \%$ \\
\hline
\end{tabular}

For each Reynolds number considered, the total vortices detected and fractions of their rotation direction are presented in the Table 1.

The figure below shows the distribution of vortices according to their range of size for each Reynolds number considered:

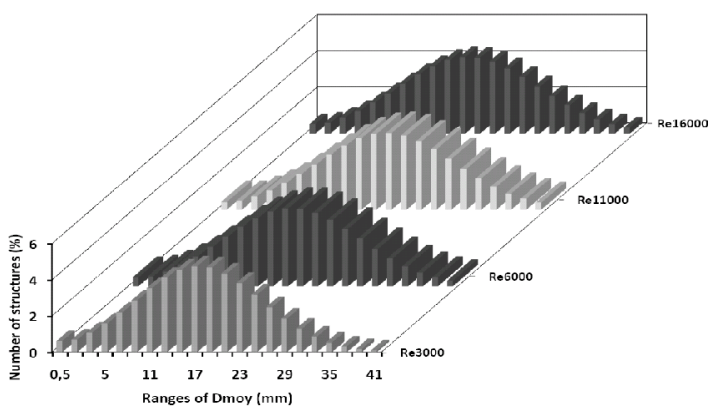

Fig. 13. Statistical analysis on mean diameter of the detected vortices at the impinging zone $(H / e=10$ and $L / e \geq 20)$

Considering the results shown on Fig. 13, the Reynolds number has a slight influence on the number of the longitudinal roll cells occurring at the impingement. Noting also that $\mathrm{Re}=6000$ seems to be a transitional value for the jet. Tsobukora et al. (2001) have predicted a similar behavior using LES and DNS simulations. A broad range of vortices coexist in the impingement region. Most of the detected vortices have their sizes between 5 and $30 \mathrm{~mm}$ (i.e. e/4 and $1.5 \mathrm{e}$ ) with the maximum situated at nearly $20 \mathrm{~mm}$ for all the Reynolds number considered.

Figure 14 shows the probability density function of eccentricity calculated for the detected vortices.

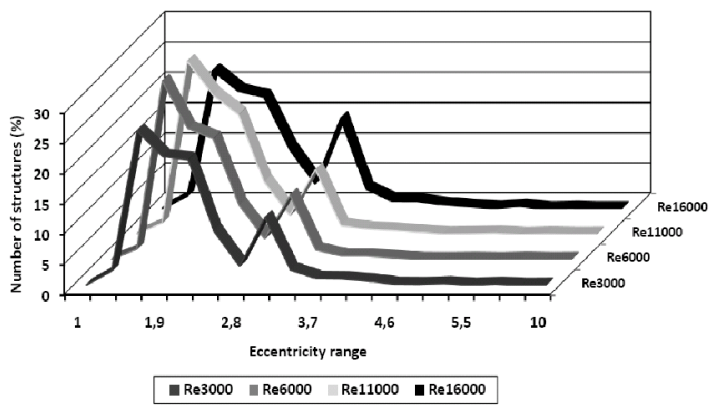

Fig.14. Probability density function of eccentricity of vortices $(H / e=10$ and $L / e \geq 20)$ 
Here results show a similarity in tendency with the results of Loubière and Pavageau (2008). We notice the presence of two distinguish maximum at 1.9 and 3.5. Most of the vortices are characterized by an eccentricity range between 1.5 and 3.7. All these findings prove that the shape of the detected vortices is elliptical.

\section{Conclusions}

The present paper focuses on the development of planar vertical impinging water jet and the flow structure in the impingement region of such flow. The potential core length is found depending on the Reynolds number and reaches a maximum of $L_{c}=3 \times e$. The evolution of the average velocity of the jet on its vertical axis is defined by the same law whatever the Reynolds number. The study was directed towards the impinging region. A characteristic height of this area $h_{i}$ was calculated. It is found slightly depending on the Reynolds number: for $\mathrm{Re}=3000$, the ratio $h_{i} / H=10 \%$ and it increases only to $13 \%$ for $\mathrm{Re}=16000$. Then, we focus on the vortex structure evolving in the impingement of the jet. A vortex educing method developed in the laboratory was used to better understand the fashion in which the large-scale structures form, evolve and contribute to transfer mechanisms. The employed algorithm is based on $\lambda_{2}$ criterion (Jeong and Hussain 1995). This criterion is used here to detect and locate the vortex core centers, the topological features of a structure being inferred from a local examination of the velocity field. It was found that:

- The Reynolds number of the flow has a slight influence on the number of vortices occurring at the impingement and their sizes,

- The shape of the detected vortices at the impingement is mainly elliptical,

- Vortex covering a wide range of sizes (from $5 \mathrm{~mm}$ to $30 \mathrm{~mm}$ ) co-exists in this region.

- The fraction of vortices having clockwise rotation direction is close to the proportion of vortices having a counter clockwise rotation direction.

\section{ACKNOWLEDGEMENTS}

This research was supported by GEPEA Laboratory and the Department of Energetic and Environmental Systems at Ecole des Mines de Nantes. These supports are gratefully acknowledged. The authors wish to thank Mrs. Yvan GOURIOU and François-Xavier BLANCHET for their help to realize our experimental arrangement.

\section{REFERENCES}

Abide S. (2005). Une méthode de décomposition de domaine pour la simulation numérique directe Contribution à l'étude de jets plans en impacts. $\mathrm{Ph}$. D. Thesis, University of Nantes, Nantes, France.

Antonia R.A., Browne L.W., Rajagopalan S., and Chambers A.J. (1983). On the organized motion of a turbulent plane jet. J. Fluid Mech. 134, 49 - 66 .

Beaubert F, and Viazzo S. (2001). Etude d'un jet plan turbulent en impact proche par simulation des grandes échelles. SFT: Transferts de chaleur et de masse dans les jets, Paris, France.

Beaubert F. (2002). Simulation des grandes échelles turbulentes d'un jet en impact. Ph. D. Thesis, University of Nantes, Nantes, France.

Beltaos S., and Rajaratnam N. (1973). Plane turbulent impinging jets. I. J. of Hydraulic Research 1, 2960.

Gardon R., and Akfirat J.C. (1965). The role of turbulence in determining the heat transfer characteristics of impinging jets. Int. J. Heat Mass Transfer 12, 1261-1272.

Gupta S. (2005). Etude expérimentale du comportement dynamique et des performances de rideaux d'air en vue de la conception de systèmes de confinement cellulaire. $\mathrm{Ph}$. D. Thesis, University of Nantes, Nantes, France.

Gupta S., and Pavageau M. (2007). Cellular confinement of tunnel sections between two air curtain. Building and Environment 42(9), $3352-$ 3365.

Gutmark E., Wolfshtein M., and Wygnanski I. (1978). The plane turbulent impinging jet. J. Fluid Mech. 88(4), 737 - 756.

Hill W.G. Jr, Jenkins R.C., and Gilbert B.L. (1976). Effect of the initial boundary layer state on turbulent jet mixing. AIAA J 17, 1055-1060.

Hussain A.K.M.F, and Clark A.R. (1977). Upstream influence on the near field of a plane turbulent jet. The Physics of Fluids 20(9), 1416-1426.

Jeong J., and Hussain F. (1995). On the Identification of a vortex. J. Fluid Mech. 285, 69-94.

Kuhlman J.M. (June 1985). Survey of near field Reynolds number effects and initial condition effects on buoyant and non-buoyant jets. Int. Symp. On Modeling Environmental Flows, Albuquerque NM, USA

Lepicovsky J. (1975). Laser velocimeter measurements of large-scale structures in a tone-excited jet. AIAA $J$ 24, 27-31.

Loubière K., and Pavageau M. (2008). Educing coherent eddy structures in air curtains systems. Chemical engineering and Processing 47, 435-448.

Lumley J. L. (1967). The structure of inhomogeneous turbulent flows. In. A. M. Ialglom\& V.I. Tatarski editors, Atmospheric Turbulence and Ratio Wave Propagation, 221-227.

Maurel S., (2001). Etude expérimentale d'un jet plan en 
A. Koched et al. / JAFM, Vol. 4, No. 2, Special Issue, pp. 61-69, 2011.

impact. Analyse paramétrique et caractérisation des transferts de masse. Ph. D. Thesis, University of Nantes, Nantes, France.

Maurel S., Rey C., Solliec C., and Pavageau M. (2004). Caractéristiques cinématiques et structurelles d'un jet d'air plan turbulent frappant une plaque plane placée à distance variable. Mécaniques \& Industries 5, 317-329.

Maurel S., and Solliec C. (2001). A turbulent plane jet impinging nearby and far from a flat plate. Experiments in Fluids 31, 687-696.

Namer I., and Ötügen MV. (1988). Velocity measurements in plane turbulent air jet at moderate numbers. Exp. Fluids 6, 387-399.

Sakakibara J., Hishida K., and Philips W.R. (2001). On the vertical structure in a plane impinging jet. $J$. Fluid Mechanics 34, 273-300.

Sakakibara, J., Hishida, K., and Maeda, M. (1997). Vortex structure and heat transfer in stagnation region of an impinging plat jet (simultaneous measurements of velocity and temperature fields by digital particle image Velocimetry and laserinduced fluorescence). Int. J. Heat and Mass Transfer 40(13), 3163-3176. 